\title{
Intelligent Monitoring Method of Short-Distance Swimming Physical Function Fatigue Limit Mobile Calculation
}

\author{
Jianxia Yin, ${ }^{1}$ Shimeng Huang $\mathbb{D},{ }^{2}$ Lei Lei $\mathbb{D}^{3},{ }^{3}$ and Jing Yao ${ }^{4}$ \\ ${ }^{1}$ Swimming Teaching and Research Office, Xi'an Physical Education University, Xi'an, 710068 Shaanxi, China \\ ${ }^{2}$ Swimming Teaching and Research Office, Guangzhou Sport University, Guangzhou, 510500 Guangdong, China \\ ${ }^{3}$ Department of Physical Education, Northwest A\&F University, Yangling, 712100 Shaanxi, China \\ ${ }^{4}$ P.E Department, Xi'an University of Technology, Xi'an, 710048 Shaanxi, China
}

Correspondence should be addressed to Shimeng Huang; 101067@tea.xaipe.edu.cn

Received 22 March 2021; Revised 16 April 2021; Accepted 13 May 2021; Published 27 May 2021

Academic Editor: Wenqing Wu

Copyright (c) 2021 Jianxia Yin et al. This is an open access article distributed under the Creative Commons Attribution License, which permits unrestricted use, distribution, and reproduction in any medium, provided the original work is properly cited.

\begin{abstract}
The detection and classification of moving targets have always been a key technology in intelligent video surveillance. Current detection and classification algorithms for moving targets still face many difficulties, mainly because of the complexity of the monitoring environment and the limitations of target characteristics. Therefore, this article conducts corresponding research on moving target detection and classification in intelligent video surveillance. According to the Gaussian Mixture Background Model and Frame Difference Method, this paper proposes a moving target detection method based on GMM (Gaussians Mixture Model) and Frame Difference Method. This method first proposes a new image combination algorithm that combines GMM and frame difference method, which solves the problems of noise and voids inside the target caused by the fusion of traditional GMM and frame difference method. The moving target detection method can effectively solve the problems of incomplete moving target detection, target internal gap, and noise, and it plays a vital role in the subsequent moving target classification process. Then, the method adds image inpainting technology to compensate the moving target in space and obtain a better target shape. The innovation of this paper is that in order to solve the multiobject classification problem, a binary tree decision support vector machine based on statistical learning is constructed as a classifier for moving object classification. Improve the learning efficiency of the classifier, solve the competitive classification problem of the traditional SVM, and increase the efficiency of the mobile computing intelligent monitoring method by more than $70 \%$.
\end{abstract}

\section{Introduction}

The rapid development of computer science and multimedia technology has driven more and more image and video applications to gradually extend to every corner of daily life. However, because the amount of information carried in images and videos is often very huge, human energy is limited after all. In some special scenes, complex and changeable harsh environments may make it difficult for humans to directly operate or cause vision due to huge workload. Fatigue, reduced work efficiency, and even operational errors lead to various unexpected and undesirable consequences. The intelligent development of society and the continuous expansion of application requirements, moving target detection and tracking algorithms with good real-time performance, high accuracy, and strong stability have become urgent needs in various fields [1]. On the basis of detailed research on the principles of various algorithms in the field of moving target detection and tracking, this article improves these two algorithms to make them have better real-time performance, thereby further improving the performance of the algorithm and improving the algorithm. Improving practicality in the field of intelligence and video survillance.

Liu Xiayu believes that based on the fusion analysis of the human body's functional characteristics, he proposed a method to monitor the natural limit of fatigue during short-distance swimming. First, a physiological indicator system was developed to monitor the natural fatigue limit of short-distance swimming, and then, feature extraction was performed to construct the natural limit of short-term 
swimmer fatigue. According to the characteristic analysis method of human physiological indicators, the monitoring characteristics of the dynamic analysis model were carried out. Monitor and, finally, monitor the functional fatigue limit of the human body in short-distance swimming. Perform machine learning and pattern recognition methods, perform short-term floating function fatigue limit monitoring, and perform data fusion analysis. However, the experiment did not carry out actual testing and lacked convincing [2]. Wang Shaohong believes that as an important part of the scientific training of competitive athletes, monitoring the physical condition of athletes is the key to supporting the scientific training of coaches. Currently, the indicators used to monitor the physical function of athletes are invasive (invasive) and noninvasive (noninvasive). The latter are mainly simple and easy-to-use noninvasive indicators, such as heart rate and weight and blood oxygen saturation. With the continuous improvement of the degree, these indicators are becoming less and less able to meet the needs of sports training. However, the algorithm is not perfect and needs to be improved [3]. Zhao Xuyang proposed a detection algorithm to determine the fatigue state of eye features and proposed a pattern recognition system based on the boundary structure learning machine to complete the tracking. When editing the facial features in this document, first, use grayscale and binary image processing, then use the KLT algorithm for face detection and positioning of the image eyes, and finally use an extreme learning machine to detect fatigue. But the system is too slow to be put into actual use [4].

Since the mobile computing intelligent monitoring method has many advantages, such as economy, convenience, and safety, compared with the traditional detection method, if it is confirmed that the mobile computing intelligent monitoring method can significantly improve the swimming ability of the swimming athletes and improve the training and competition performance, then this monitoring method will. It is a good choice for endurance sports to improve athletic ability. The influence on the athletic ability of swimmers is the most important indicator for evaluating this training method $[5,6]$. This article attempts to reveal the influence of short-distance swimming physical function fatigue limit on swimmers' athletic ability through comparative experiments, analyze the mechanism of its effect on the body, evaluate the training effects, and propose scientific training methods [1].

\section{Mobile Computing Intelligent Monitoring Method}

2.1. Interframe Difference Method. When a foreground target appears in a video sequence, the images of the two frames before and after it will change significantly. The frame difference method uses this function to subtract the video sequence frame by frame to obtain the brightness difference $[2,7]$. To determine whether the current frame contains foreground objects, you need to compare the difference with the set threshold. The frame difference method has low algorithm complexity, good real-time performance, and strong robustness for image sequences with relatively clear fore- ground target contours. However, this algorithm cannot accurately identify a complete foreground target and can only extract the approximate outline of the target, thereby forming a large number of holes in the foreground area. And for images with blurred target contours, it is often impossible to achieve better detection results $[8,9]$.

2.2. Background Subtraction. In order to solve the problem of the frame difference method, Glober et al. used a prospect extraction method combined with mathematical statistics. First, the median method is used to construct a background sample set for the image, that is, the median value of the pixel in the adjacent frames is obtained for sampling. Then, the newly arrived image and the sample set are subjected to a different operation to extract the foreground pixels. Although this algorithm can overcome the problem that the frame difference method can only detect the target contour, its time efficiency and space efficiency are not high, and large detection errors are often prone to occur in video sequences with unstable lighting conditions $[10,11]$. In order to overcome this problem, Wren et al. introduced the Gaussian model to improve the sample modeling and set a threshold to classify the pixels.

2.3. Other Target Detection Methods. The development of background subtraction has gone through a process from simple to complex and, then, from complex to simplification $[12,13]$. While the background subtraction method continues to improve, other target detection algorithms with different principles have gradually entered people's sight, such as target detection algorithm combined with fuzzy theory, target detection algorithm combined with motion estimation, and target detection algorithm combined with neural network. In order to solve the problem of low accuracy and poor stability in the detection process of some background subtraction methods $[14,15]$, some researchers have proposed a method that combines target detection algorithms with fuzzy theory. The experimental results do verify that this type of algorithm has high accuracy and stability, but its time and space efficiency do not have good real-time performance under current hardware conditions [16].

\section{Correlation Experiment of Moving Target Detection and Classification Algorithm}

In computer image processing and analysis, moving target detection technology has always been a research hotspot in video sequence analysis. The so-called moving object detection is the process of separating moving objects from the background image of the video. Due to the complex background, illumination changes, and severe weather in the real scene, the detection of moving objects still faces huge challenges $[17,18]$.

3.1. Optical Flow Method. Assume that in the case of $n$ dimensions (the same applies to 3D and higher dimensions) after time $t$ and time $t$, the gray value of the pixel $(x, y)$ of the video frame is $f(x, y)$, and the pixel is at move $x 1$ in $x$ direction and $y 1$ in $y$ direction. The pixel value corresponding to 
the pixel at this moment is $f(x+x 1, y+y 1)$ [19]. Assuming that $t$ is small, the gray value of the pixel will remain unchanged in a very short time, so formula (1) can be used to express that the pixel values of the two points are equal.

$$
f(x, y, t)=f(x+x 1, y+y 1, t+t 1)
$$

Since the displacement is very small, the Taylor expansion of equation (1) is performed to obtain the equation $[20,21]$ :

$$
f(x+x 1, y+y 1, t+t 1)=f(x, y, t)+\sigma^{2} .
$$

Among them, $\partial$ is the partial derivative of $f(x, y, t)$ with respect to $x, y, t$ of the second order and above, which can be ignored, so formula (3) can be obtained:

$$
\frac{d f}{d x} x+\frac{d f}{d y} y+\frac{d f}{d t} t=0 .
$$
(4):

Divide both sides of the equation by $t$ to obtain equation

$$
\frac{d f}{d x} \frac{d x}{d t}+\frac{d f}{d y} \frac{d y}{d t}+\frac{d f}{d t} \frac{d t}{d t}=0
$$

When $t$ approaches 0 , there are $[22,23]$ :

$$
\frac{d f}{d x} W_{x}+\frac{d f}{d y} V_{y}+\frac{d f}{d \mathrm{t}}=0 .
$$

The vector sum of $W$ and $V$ is the luminous flux of a certain pixel in the video image. Equation (5) is called the optical flow constraint equation.

3.2. Frame Difference Method. The frame difference method uses the difference operation of two or three consecutive frames in the video sequence and uses the time difference to extract the moving area in the image, thereby obtaining the moving target. Figure 1 shows the effect of the frame difference method on moving target detection.

$f(x, y, t), f(x, y, t-1)$ represent the current frame and the previous frame of the current frame, respectively, assuming $D(x, y, t)$ is the difference between the current frame and the previous frame. The result can be expressed by equation (6) $[23,24]$ :

$$
D(x, y, t)=f(x, y, t)-f(x, y, t-1)
$$

where $(x, y)$ represents the position of the pixel $R(x, y)$, $R x y k$ represents the image after binarization processing, $R(x, y)$ takes 1 to indicate that the current pixel is a moving target, $R(x, y)$ takes 0 , which indicates that the current pixel is the background, and Trepresents the threshold $[25,26]$.

$$
R(x, y)=\left\{\begin{array}{ll}
1 & D(x, y) \geq T \\
0 & D(x, y) \leq T
\end{array} .\right.
$$

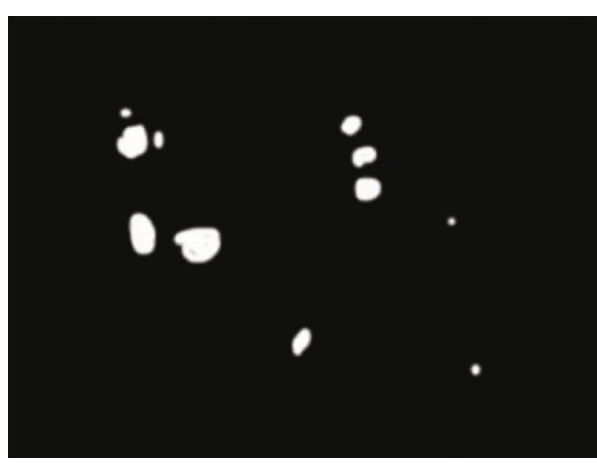

Figure 1: Examples of moving target detection pictures.

TABLE 1: The dynamic changes of intermittent hypoxia training on athletes' RBC.

\begin{tabular}{lcc}
\hline & Test group & Control group \\
\hline Before the experiment & 4.54 & 4.52 \\
Day 4 & 4.66 & 4.50 \\
Day 8 & 4.80 & 4.52 \\
Day 11 & 4.86 & 4.57 \\
Day 15 & 4.79 & 4.48 \\
Day 18 & 4.68 & 4.42 \\
Day 21 & 5.10 & 4.65 \\
\hline
\end{tabular}

The frame difference method is simple to implement, does not require background modeling and updating, the algorithm is fast, is not sensitive to changes in lighting, and can adapt to complex and changeable environments, and is suitable for scenes with high real-time requirements. However, the frame difference method cannot completely extract the moving target; only the contour information can be extracted, so the target information is incomplete. If the target feature is subsequently extracted, the feature information will be incomplete [27].

\section{Short-Distance Swimming Physical Function Fatigue Limit Analysis}

4.1. Effect of Intermittent Hypoxia Training on Swimmers. In this study, the serum ferritin of the athletes in the experimental group and the control group was in the normal range before and after the experiment, and there was no significant difference, indicating that the two groups did not decrease serum ferritin due to a lot of training. Iron is the raw material for red blood cell production, and serum ferritin is an indicator for detecting human iron reserves.

It can be seen from Table 1 that before the start of the experiment, there was no significant difference in the red blood cell counts of the athletes in the experimental group and the control group, and the two groups were comparable; in the simulated hypoxic environment caused by living high and training low, the red blood cell counts of the athletes in the experimental group were short-term. Although there was an increase in internal, it did not reach statistical significance. Until the end of the experiment, the number of red 


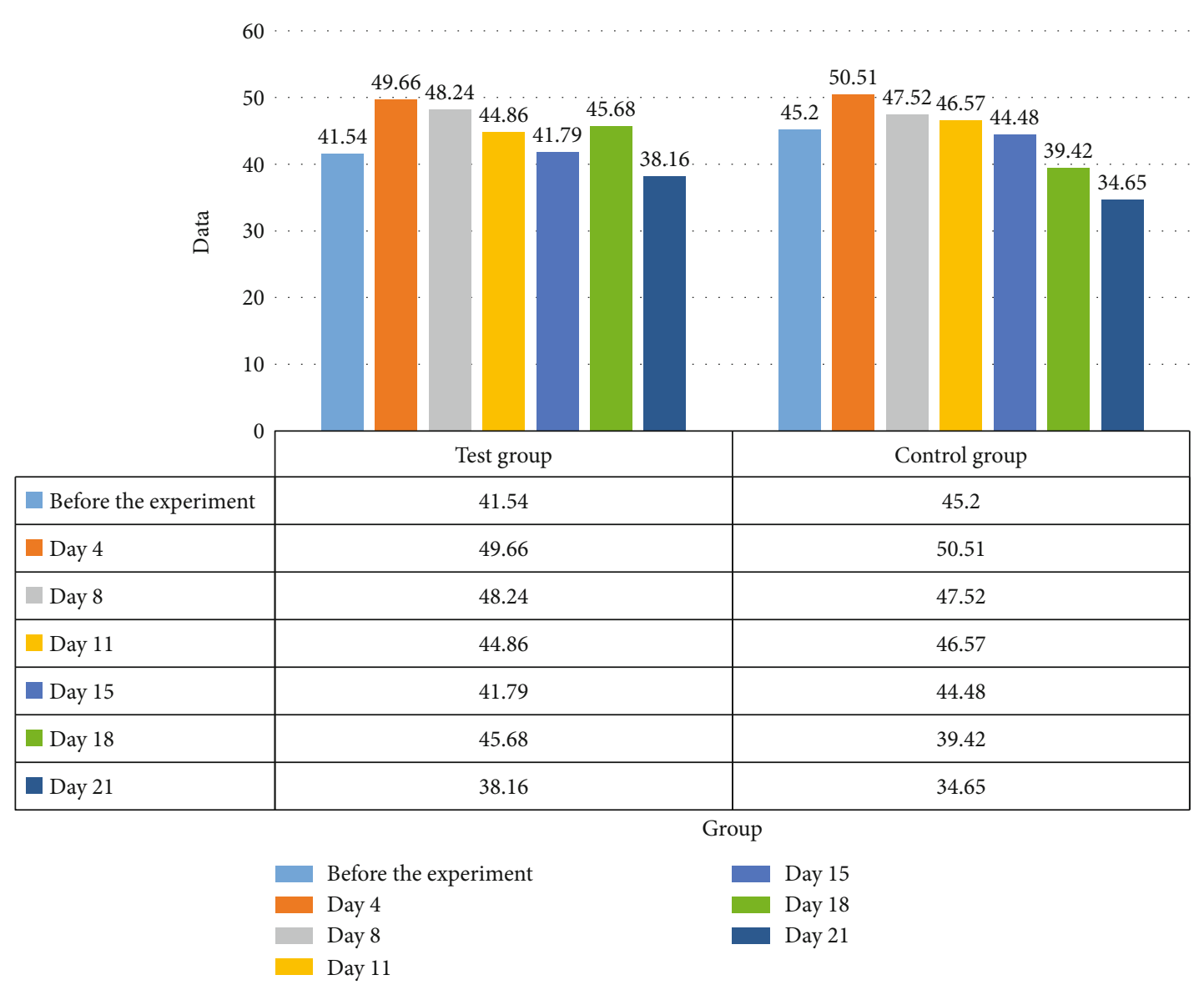

FIGURE 2: The dynamic changes of HCT of athletes during intermittent hypoxia training.

blood cells increased significantly compared to before the experiment. There was no significant change in the number of red blood cells of the athletes in the control group before and after the experiment. It shows that the training method of intermittent hypoxic training can significantly increase the red blood cell count and enhance the body's oxygencarrying capacity, but this effect must be displayed after about 3 weeks of training, so pay attention to intermittent hypoxic training time control. Since the life span of normal red blood cells in the human body is 120 days, after the athlete returns to normal training after the intermittent hypoxic training, the high level of RBC will remain for a period of time.

As shown in Figure 2, before the start of the experiment, there was no significant difference in hematocrit between the test group and the control group, and the two groups were comparable. In the simulated hypoxic environment caused by living high and training low, the hematocrit of the experimental group had a significant increase on the 8th day, and it continued to reach the highest value at the end of the experiment. There was no significant change in the hematocrit of the control group athletes before and after the experiment. Within a certain range, the hematocrit is directly proportional to the blood's oxygen-carrying capacity, that is, the larger the HCT, the stronger the blood's oxygen-carrying capacity; however, when the HCT exceeds a certain range (generally considered to be more than $50 \%$ ), the blood is
TABLE 2: Weight measurement values before and after intermittent hypoxia training.

\begin{tabular}{|c|c|c|c|}
\hline & $\begin{array}{l}\text { Test } \\
\text { group }\end{array}$ & $\begin{array}{l}\text { Control } \\
\text { group }\end{array}$ & $\begin{array}{l}\text { Experimental group } \\
\text { compared with control }\end{array}$ \\
\hline $\begin{array}{l}\text { Before the } \\
\text { experiment } 1\end{array}$ & 67.08 & 63.38 & $P>0.05$ \\
\hline $\begin{array}{l}\text { Before the } \\
\text { experiment } 2\end{array}$ & 66.37 & 63.42 & $P>0.05$ \\
\hline In experiment 1 & 66.39 & 63.33 & $P>0.05$ \\
\hline In experiment 2 & 66.20 & 63.17 & $P>0.05$ \\
\hline $\begin{array}{l}\text { After the } \\
\text { experiment }\end{array}$ & 65.29 & 63.43 & $P>0.05$ \\
\hline
\end{tabular}

sticky. Sexual enhancement affects blood rheology. Experiments show that the HCT of all athletes is in the best range, indicating that the effective oxygen uptake of the athletes' "red blood cells" is in the best state.

4.2. Effect of Intermittent Hypoxia Training on the Body Water of Swimmers. The stable state of human physiological processes relies on the close coordination of nerves and body fluids. Generally speaking, nerve regulation is rapid and short-lived, while body fluid regulation is slow and long. The two complement each other and are in harmony. 


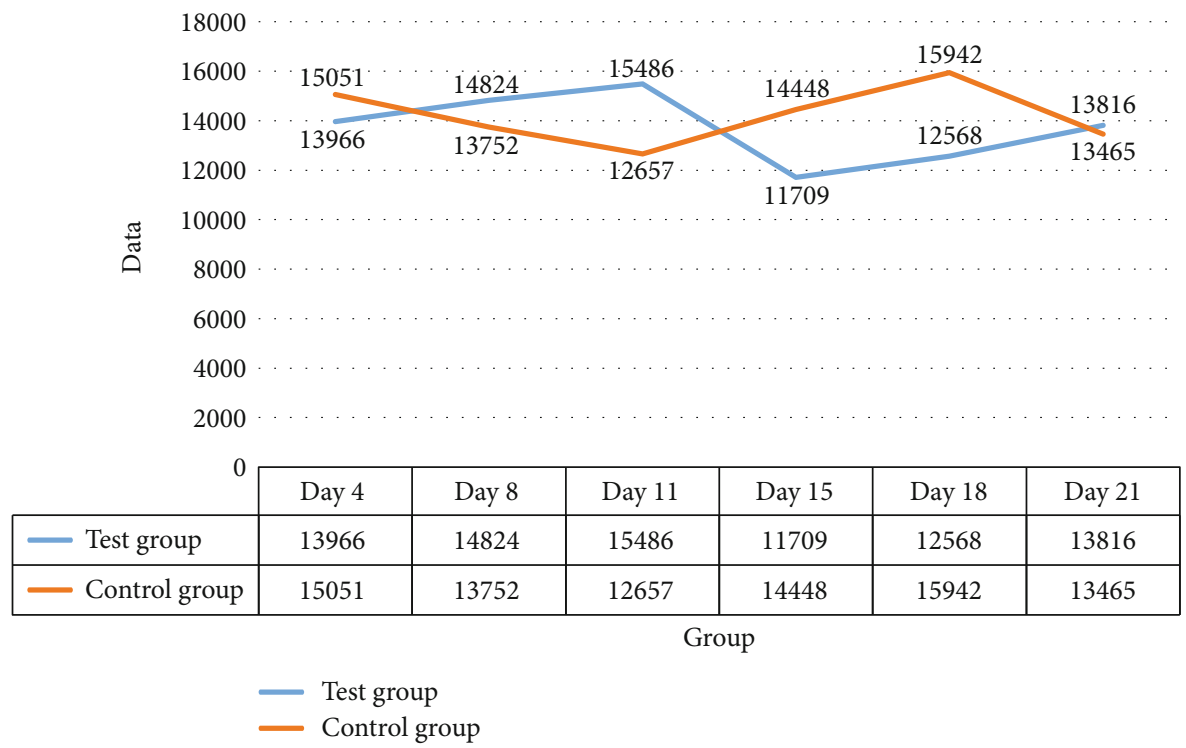

Figure 3: Control of exercise load of swimmers during intermittent hypoxic training.

It can be seen from Table 2 that the weight of athletes in the experimental group before intermittent hypoxic training is not significantly different from that of the control group $(P>0.05)$, indicating that the two groups are comparable; tested in the experiment and after the experiment, the experimental group had no significant difference between the athletes' weight and the control group $(P>0.05)$. There was no significant difference in weight before and after the experiment in the control group athletes. The weight of the athletes in the experimental group decreased significantly after the experiment compared with before the experiment $(P<0.05$ ) , indicating that the athletes' weight decreased significantly after the intermittent hypoxic training.

As can be seen from Figure 3, the amount of exercise curve goes from low to high and, then, from high to low. The exercise volume in the first two weeks is higher, which is to adapt to the exercise load stimulation and hypoxic stimulation to make the athlete's body produce and improve the exercise adaptability. At 15 days, the red blood cell count and hemoglobin showed a downward trend, indicating that the athletes had accumulated a certain degree of fatigue after two weeks of training. Therefore, in the third week, the amount of exercise decreased and the exercise intensity increased moderately.

\section{Conclusions}

This paper combines the idea of background difference in the target detection algorithm to realize the automatic initialization of the search box. Aiming at the problem that the Camshift algorithm is easy to lose the target when the lighting conditions change drastically, a judgment strategy of the average value and the actual value of the Hue component is introduced to realize the real-time update of the color probability model. Aiming at the problem of tracking failure of the Camshift algorithm after the target moves at a high speed or is temporarily occluded, this paper combines the Kalman filter algorithm to introduce a motion prediction mechanism, which predicts the possible position of the target in the next frame during the tracking process. Experimental results show that the improved Camshift algorithm can maintain a good tracking effect even under sudden changes in lighting. In addition, when the target moves at a high speed or is temporarily occluded, the improved algorithm can still maintain a stable recognition rate. This paper proposes an improved strategy for the deficiencies of the ViBe target detection algorithm and the Camshift target tracking algorithm, and the effectiveness of the improvement is verified through experiments. However, there are still some unresolved problems in the research process. Therefore, the realization of adaptive sharpening of the target area will be the focus of future research. It is also in the future to extend the improved Camshift algorithm to the application of multitarget tracking, a new direction of work.

\section{Data Availability}

The data that support the findings of this study are available from the corresponding author upon reasonable request.

\section{Conflicts of Interest}

The authors declare that they have no conflicts of interest.

\section{References}

[1] L. Xiayu and N. Zhebin, "Research on the limit monitoring method of physical function fatigue in short-distance swimming," Journal of Chifeng University (Natural Science Edition), vol. 35, no. 300, pp. 107-110, 2019.

[2] W. Shaohong, L. Weiguo, N. Yuhua, and W. Chunjian, "Exercise load monitoring and fatigue evaluation, recovery and nutritional supplementation of middle and short distance swimmers," Contemporary Sports Science and Technology, vol. 9, no. 21, pp. 28-29, 2019. 
[3] Z. Xuyang and Y. Wang, "Research on fatigue driving monitoring method based on extreme learning machine face recognition," China Equipment Engineering, no. 9, pp. 85-86, 2018.

[4] Y. Liang, X. Hu, and Q. Yang, "Research on intelligent recommendation algorithm for mobile services based on group intelligence computing," Digital Technology and Application, vol. 38, no. 358, pp. 119-120, 2020.

[5] B. Yuanyuan, "Analysis and research on fatigue characteristics of swimming training," Stationery \& Sports Supplies \& Technology, no. 21, pp. 214-215, 2019.

[6] L. Qing and Y. Qiang, "Short-distance swimming squatting start technical characteristics and special training," Journal of Capital Institute of Physical Education, vol. 29, no. 6, pp. 549-553, 2017.

[7] Z. Qixiang, "Research on the structure and level of the special ability of short-distance swimmers," Sports Time and Space, no. 23, pp. 96-97, 2017.

[8] J. Chengping, S. Yunyun, and H. Xinzhong, "The control of Yu Hexin's multi-year training arrangements for outstanding short-distance swimmers," Journal of Beijing Sport University, vol. 41, no. 11, pp. 109-115, 2018.

[9] L. Jianxun, "A preliminary study on the short-distance special ability training of swimmers aged 6-12 years," Leisure, no. 7, pp. 89-89, 2019.

[10] Z. Jie, L. Yijun, Z. Xiaodong, and K. Dejia, "Study on stroke frequency, stroke width and speed of excellent female $200 \mathrm{~m}$ swimmers during competition," Journal of Xi'an Institute of Physical Education, vol. 33, no. 1, pp. 124-128, 2016.

[11] Y. Tian, L. Zhang, H. Wang et al., "Intelligent detection platform for simultaneous detection of multiple MiRNAs based on smartphone," ACS Sensors, vol. 4, no. 7, pp. 1873-1880, 2019.

[12] S. Yang and L. Ying, "Research on winning factors of $50 \mathrm{~m}$ short-distance swimming competition," Contemporary Sports Science and Technology, vol. 7, no. 32, pp. 160-161, 2017.

[13] L. Mingguang, "The importance and methods of strength training for short-distance swimmers," Journal of Science Education (Electronic Edition), no. 2, pp. 103-103, 2017.

[14] Z. Yong, "Monitoring and analysis of biochemical indicators of outstanding long and short distance female swimmers," Chinese Sports Coaches, vol. 26, no. 4, pp. 74-75, 2018.

[15] W. Youhuai, "Discussion on the pre-match training of shortdistance high-level swimmers in Guangdong Province," Sports Fashion, no. 11, pp. 36-37, 2019.

[16] J. Zhixiang, "Research on the methods of strength training for short-distance swimming athletes," Contemporary Sports Science and Technology, vol. 6, no. 28, pp. 57-58, 2016.

[17] S. Wen and C. Jingjing, "Analysis of the stroke time of men's short-distance freestyle swimmers of Guangdong swimming team," Contemporary Sports Science and Technology, vol. 6, no. 10 , pp. $138-140,2016$.

[18] G. Lei, "Research on the stroke technique of men's shortdistance freestyle swimmers," Knowledge Library, no. 6, pp. 196-197, 2019.

[19] Б. П. Yakovlev, Г. Д. Babshikin, Р. E. Lou Bin, Е. Г. Babshikin, И. Б. Taraschenko, and Y. Songping, "Special training research on swimmers in the pre-competition and mid-cycle period," Journal of Capital Institute of Physical Education, vol. 30, no. 4, pp. 289-291, 2018.
[20] M. Xiaochen, H. Yu, and R. Chen, "Research on effective landbased intensity exercise methods for $100 \mathrm{~m}$ swimming events," Contemporary Sports Science and Technology, vol. 7, no. 2, pp. 249-252, 2017, 254.

[21] Z. Wenxiu, "Research on physical training methods and characteristics of short, middle and long distance swimming events," Contemporary Sports Science and Technology, vol. 9, no. 35, pp. 27-28, 2019, 30.

[22] L. Dongyu and Z. Deshan, "Bivariate analysis of Chinese and foreign outstanding men's $50 \mathrm{~m}$ breaststroke," Contemporary Sports Science and Technology, vol. 8, no. 20, pp. 235-236, 2018.

[23] L. Dongyu and L. Wenmin, "Using Pearson correlation to analyze the effect of stroke frequency on the performance of 50meter breaststroke," Guizhou Sports Science and Technology, no. 2, pp. 46-48, 2018.

[24] Y.-S. Choi, J.-H. Hong, and J.-M. Woo, "Design of Antenna for intelligent detection sensor," The Journal of The Korea Institute of Intelligent Transport Systems, vol. 19, no. 2, pp. 104109, 2020.

[25] Z. Xiaofeng, "Discussion on the explosive training methods of upper limbs of swimmers," Contemporary Sports Science and Technology, vol. 8, no. 5, pp. 38-39, 2018.

[26] L. Taibo, "Research on the swimming training mode of the seamen's skill competition," Sports Boutique (Academic Edition), vol. 36, no. 11, pp. 83-84, 2017.

[27] L. Hai, W. Yongxue, Q. Jinjin et al., "Niche and interspecies encounter probability of main swimming animals in Yiwan Bay, Taizhou," Chinese Journal of Fishery Sciences, vol. 26, no. 2, pp. 136-144, 2019. 